\title{
Effects of Adolescent Cannabinoid Self-Administration in Rats on Addiction-Related Behaviors and Working Memory
}

\author{
Erin K Kirschmann', Michael W Pollock', Vidhya Nagarajan' and Mary M Torregrossa*,1,2 \\ 'Department of Psychiatry, University of Pittsburgh, Pittsburgh, PA, USA; ${ }^{2}$ Center for Neuroscience, University of Pittsburgh, Pittsburgh, PA, USA
}

\begin{abstract}
Use of marijuana (Cannabis sativa) often begins in adolescence, and heavy adolescent marijuana use is often associated with impaired cognitive function in adulthood. However, clinical reports of long-lasting cognitive deficits, particularly in subjects who discontinue use in adulthood, are mixed. Moreover, dissociating innate differences in cognitive function from cannabis-induced deficits is challenging. Therefore, the current study sought to develop a rodent model of adolescent cannabinoid self-administration (SA), using the synthetic cannabinoid receptor agonist WIN55,2I2-2 (WIN), in order to assess measures of relapse/reinstatement of drug seeking and long-term effects on cognitive function assessed in a delay-match-to-sample working memory task and a spatial recognition task. Adolescent male rats readily self-administered WIN in 2-h or 6-h sessions/day, but did not demonstrate an escalation of intake with 6-h access. Rats exhibited significant cue-induced reinstatement of WIN seeking that increased with 21 days of abstinence (ie, 'incubation of craving'). Cognitive testing occurred in adulthood under drug-free conditions. Both 2-h and 6-h adolescent WIN SA groups exhibited significantly better working memory performance in adulthood relative to sucrose SA controls, and performance was associated with altered expression of proteins regulating GABAergic and glutamatergic signaling in the prefrontal cortex. Self-administered WIN did not produce either acute or chronic effects on short-term memory, but experimenter administration of WIN in adolescence, at doses previously reported in the literature, produced acute deficits in short-term memory that recovered with abstinence. Thus, SA of a rewarding cannabinoid in adolescence does not produce long-term cognitive dysfunction.

Neuropsychopharmacology (2017) 42, 989-1000; doi: 10.1038/npp.2016.178; published online 5 October 2016
\end{abstract}

\section{INTRODUCTION}

Marijuana (Cannabis sativa) is the most commonly used illicit substance in the United States, and initiation of use in adolescence is common (Moss et al, 2014). Cannabis use, particularly in adolescence, is frequently associated with negative long-term functional outcomes. For example, reduced IQ, increased risk for schizophrenia, and impaired working memory have all been associated with adolescent cannabis use (Becker et al, 2014; Gage et al, 2015; Marconi et al, 2016; Meier et al, 2012). However, a direct causal link between cannabis use and cognitive impairments has not been definitively demonstrated. In most studies, it has been impossible to control for pre-existing differences in cognitive capabilities, genetics, or for use of other substances (Hooper et al, 2014; Rogeberg, 2013). Moreover, several clinical studies have failed to find differences between cannabis users and controls on cognitive measures (Buchy et al, 2015; Jager et al, 2006; Mokrysz et al, 2016). Now, with the increasing legalization of marijuana, medicinal use, and perception of safety, it is expected that the use of cannabis and

\footnotetext{
*Correspondence: Dr MM Torregrossa, Department of Psychiatry, University of Pittsburgh, Bridgeside Point II, Rm 228, 450 Technology Dr, Pittsburgh, PA 15219, USA, Tel: + I 4I2 624 5723, Fax: + I 412624 5280, E-mail: torregrossam@upmc.edu

Received 13 May 2016; revised 5 July 2016; accepted 22 July 2016; accepted article preview online I September 2016
}

cannabinoid-related compounds in adolescence will continue to rise (Miech et al, 2015). Therefore, it is critical to increase our understanding of both cannabinoid addiction and the consequences of cannabinoid use on cognitive and emotional function in adolescence.

To date, there has been limited use of translational models for understanding cannabinoid abuse liability, mechanisms of addiction, or effects of self-administered dosing patterns on cognition. Moreover, studies of adolescent selfadministration (SA) of cannabinoids are completely lacking. One reason for the paucity of pre-clinical research is that the primary psychoactive constituent of marijuana, $\Delta 9$-tetrahydrocannabinol (THC) is not readily self-administered by rodents (Takahashi and Singer, 1979). However, the synthetic cannabinoid receptor agonist, WIN55,212-2 (WIN), which is a more potent agonist at cannabinoid receptors (CB) 1 and 2 than THC, is self-administered by rats (Fattore et al, 2001; Justinova et al, 2013; Lefever et al, 2014). The effects of WIN may be more comparable to the frequently abused synthetic cannabinoids, often referred to as K2 and spice, than to marijuana (Gunderson et al, 2012; Zawilska and Wojcieszak, 2014), but use of WIN can clarify the effects of cannabinoid receptor agonist SA on behavior. Moreover, in rodents, short-term memory deficits in spatial, novel object, and social recognition are produced by administration of either THC or WIN, and memory deficits are prevented by administration of nonselective $\mathrm{CB}$ receptor 
antagonists (Abush and Akirav, 2012; O'Shea et al, 2004, 2006; Renard et al, 2016; Schneider and Koch, 2003; Wiley and Burston, 2010). Therefore, any effects of THC on cognition are likely to be recapitulated by WIN. However, the cognitive effects of doses self-administered by animals are unknown. Therefore, the present study established WIN $\mathrm{SA}$ in adolescent, male Sprague Dawley rats to determine the long-term effects of adolescent WIN SA on adult cognitive function and addiction-related behaviors. Moreover, owing to the known involvement of the prefrontal cortex (PFC) in working memory performance, particularly excitatory-inhibitory balance in the PFC (Cohen Kadosh et al, 2015; Compte et al, 2000; Michels et al, 2012), we confirmed PFC involvement in our working memory task and assessed effects of WIN SA on PFC GABAergic and glutamatergic protein expression.

\section{MATERIALS AND METHODS}

\section{Animals}

A total of 97 male, Sprague Dawley rats (Harlan, Indianapolis, IN) delivered on postnatal day (PD) 22-30 were housed in a climate-controlled room on a $12 \mathrm{~h}$ light/dark cycle (lights on at 4:30 am) for all experiments. Rats were pair-housed with ad libitum access to food and tap water, except for periods of food restrictions described below. All procedures were approved by the University of Pittsburgh Institutional Animal Care and Use Committee, and were performed in accordance with the National Institutes of Health Guide for the Care and Use of Laboratory Animals.

\section{Drugs}

The synthetic cannabinoid receptor agonist WIN55,212-2 mesylate (WIN; provided by the National Institutes of Mental Health Chemical Synthesis and Drug Supply Program) was dissolved in sterile $0.9 \%$ saline with a drop of Tween 80. Fresh stock solutions were prepared every 2-3 days, and were diluted daily with saline for intravenous (IV) and intraperitoneal (IP) administration. The $\mathrm{GABA}_{\mathrm{A}}$ receptor agonist muscimol and $\mathrm{GABA}_{\mathrm{B}}$ receptor agonist baclofen (Sigma-Aldrich, St Louis, MO) were dissolved in sterile saline.

\section{Surgical Procedures}

Surgeries were performed as described previously (Torregrossa and Kalivas, 2008; Torregrossa et al, 2010, 2013). In brief, rats (PD25 or 28) were anesthetized with ketamine $(87.5 \mathrm{mg} / \mathrm{kg})$ and xylazine $(5 \mathrm{mg} / \mathrm{kg})$, given $5 \mathrm{mg} / \mathrm{kg}$ of the analgesic Rimadyl (Henry Schein, Dublin, $\mathrm{OH})$, and were implanted with indwelling jugular catheters (CamCaths, Cambridge, UK). Controls received identical surgical procedures. A separate group of adult rats with previous adolescent working memory training $(n=16)$ received bilateral intracranial cannulae (22 gauge; Plastics One, Roanoke, VA) aimed at the prelimbic (PL) PFC using the following coordinates (relative to bregma: AP $+3.2 \mathrm{~mm}$, $\mathrm{ML} \pm 0.75 \mathrm{~mm}, \mathrm{DV}-3.2 \mathrm{~mm}$ (Paxinos and Watson, 2005).

\section{Cannabinoid Self-Administration}

Rats were food restricted (15-20g rat chow per day) to maintain $85-90 \%$ of their free-feeding weight and trained to self-administer the synthetic cannabinoid agonist WIN $(0.0125 \mathrm{mg} / \mathrm{kg} /$ infusion $)$ in standard operant conditioning chambers (Med Associates, St Albans, VT). The chambers contained two retractable levers, a food magazine, a house light, two stimulus lights, a tone generator, and a fan for background noise. The dose for SA was selected based on published studies (Fattore et al, 2001; Justinova et al, 2013). Rats were trained on a fixed-ratio 1 (FR1) schedule of reinforcement in daily 1-h sessions for 4 days, and then were switched to either 2-h (short-access; $n=12$ ) or 6-h (longaccess; $n=12$ ) SA sessions (see Figures $1 \mathrm{a}$ and $3 \mathrm{a}$ ). Rats were randomly assigned to an active lever (counterbalanced across groups), and inactive lever responses were recorded, but had no consequences. Each infusion of WIN was paired with a $10 \mathrm{~s}$ light/tone-conditioned stimulus and $10 \mathrm{~s}$ timeout. Control rats $(n=12$ /group) followed identical training procedures but responded for sucrose pellets $(45 \mathrm{mg}$; BioServ, Flemington, NJ). Long-access rats were tested for cueinduced reinstatement after 8 and 29 days of abstinence to test for relapse-like behavior and incubation of craving. These rats had 7 days of 1 -h lever extinction training, in which active lever responses did not result in a reinforcer or cue presentation. Reinstatement to response-contingent cue presentations was tested in 30-min sessions on PD69 and PD90 (Figure 3a).

\section{Experimenter-Administered Cannabinoids}

A separate group of rats received IP injections of vehicle $(n=17)$, or WIN $(1.2 \mathrm{mg} / \mathrm{kg} ; n=16)$ daily throughout adolescence (PD34-54; Figure 5a). Rats went through a washout period from PD55-71 before working memory training. The WIN dose was chosen based on what has been reported in the literature to produce short-term memory impairments (O’Shea et al, 2006; Schneider and Koch, 2003).

\section{Delay-Match-to-Sample Working Memory Task}

After SA, rats had $a \geqslant 10$ day washout period where they remained in their homecage before working memory training ( $\geqslant$ PD70). Rats were trained in daily 1 -h sessions in chambers (Med Associates) equipped with five nosepoke apertures, food dispenser, and a fan for background noise. Rats were initially trained to poke into any aperture for a sucrose pellet on an FR1 schedule. Next, a response on a single lighted 'sample' aperture resulted in immediate illumination of three 'choice' apertures. A second response in the sample aperture resulted in sucrose reward (FR1). Once rats reliably responded twice in the correct aperture ( $>75 \%$ accuracy), delays were introduced between the sample and the choice phases. Rats performed blocks of trials in which seven delays $(0.5-6 \mathrm{~s})$ were presented in random order; each of the seven delays occurred before a new block of trials began. Once rats reached training criterion ( $\geqslant 80 \%$ correct $0.5 \mathrm{~s}$ delay), the range was increased (0.5-12s; 0.5-24s) in order to assess working memory capacity. The rats' behavior was occasionally monitored to ensure movement away from apertures during delay periods. 
a

\begin{tabular}{|c|c|c|c|c|c|c|}
\hline PD25 & PD28 & PD38-49 & PD50-86 & PD87-109 & PD110-118 & \\
\hline \multirow[t]{2}{*}{ Arrive } & \multirow{2}{*}{$\begin{array}{c}\text { IV } \\
\text { Surgery }\end{array}$} & \multirow{2}{*}{$\begin{array}{c}\text { WIN55,212-2 or Sucrose SA } \\
\text { Short-access (2hr) }\end{array}$} & Washout & \multirow{2}{*}{$\begin{array}{l}\text { WM Task } \\
\text { Training }\end{array}$} & \multirow{2}{*}{$\begin{array}{c}\text { WM } \\
\text { Assessment }\end{array}$} & Collection \\
\hline & & & & & & \\
\hline
\end{tabular}
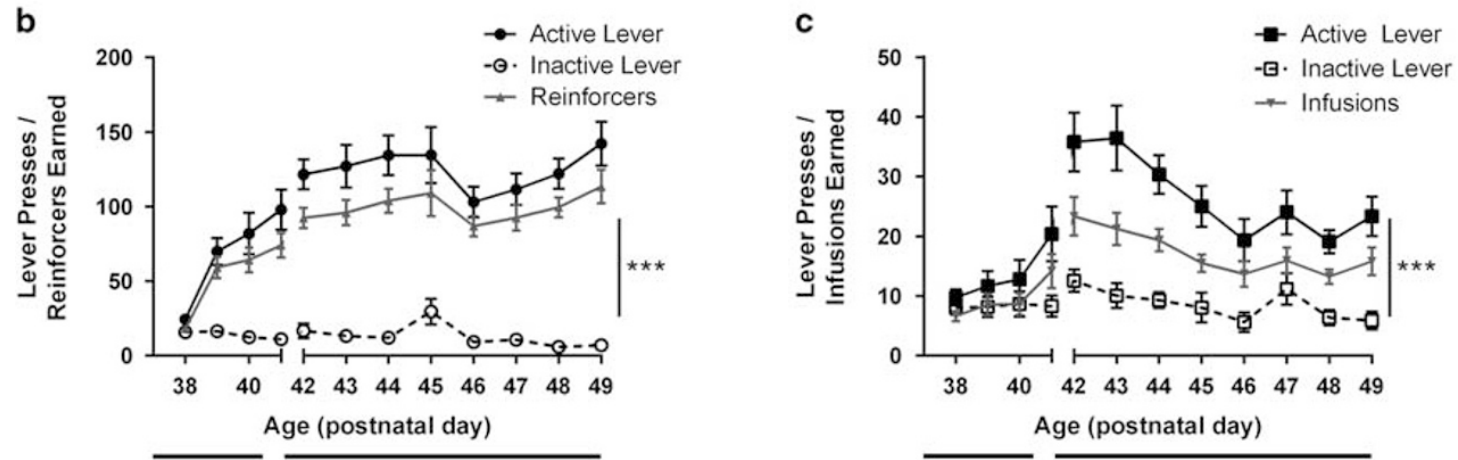

$1 \mathrm{hr}$ sucrose SA 2hr sucrose SA

$1 \overline{\mathrm{hr} \text { WIN SA }} \quad$ 2hr WIN SA
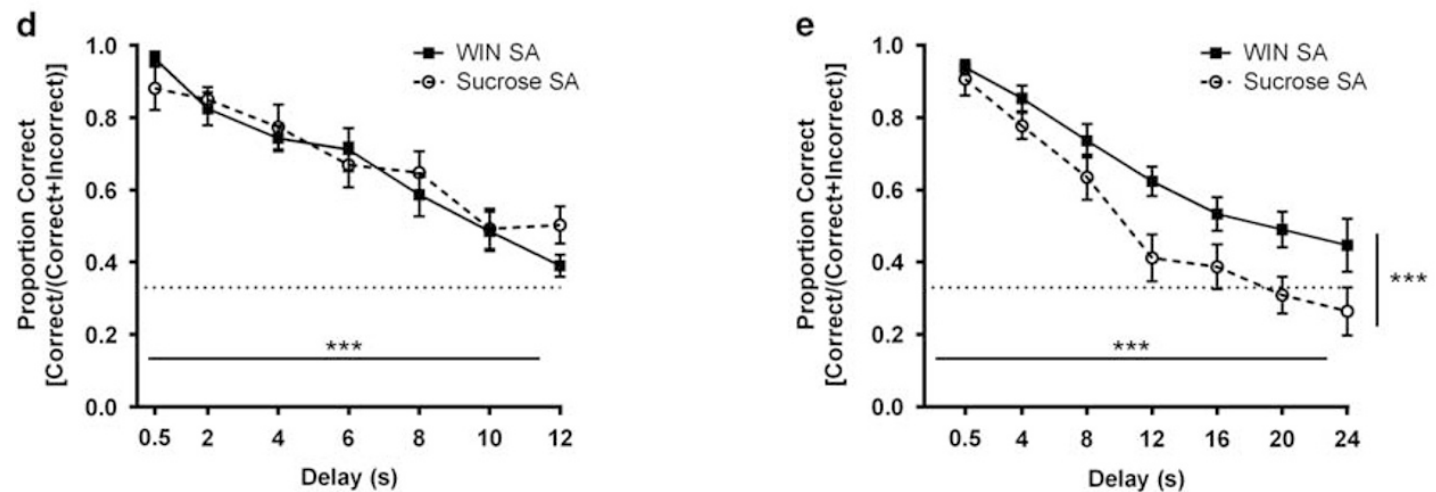

Figure I Short-access cannabinoid self-administration during adolescence improves working memory performance in adulthood. (a) Experimental timeline. Rats ( $n=12$ per group) were trained to self-administer (b) sucrose pellets or (c) WIN55,212-2 (0.0 I25 mg/kg/infusion). Active lever presses were significantly higher than inactive in every 2-h session for both sucrose and WIN groups. Reinforcers or infusions earned are shown in gray in (b) and (c), respectively. (d) Working memory performance did not differ between self-administration groups during training on $0-12 \mathrm{~s}$ delays. (e) Rats with adolescent WIN selfadministration experience exhibited better working memory when the delay range was expanded to $0-24 \mathrm{~s}$. The proportion of correct responses for WIN SA rats was greater than sucrose SA controls across many delays with high cognitive load. Values show group means \pm SEM; IV: intravenous; PD: postnatal day; SA: self-administration; WM: working memory; **** $p<0.001$.

In addition, if a rat remained seated in front of the original sample aperture, its positioning would be such that it remained in front of all three choice apertures as well, thus requiring the rat to maintain sample information in working memory during the delay. Finally, reaction times for latency to perform correct/incorrect trials were recorded.

\section{Drug Infusions}

In order to confirm that our working memory task required functional prefrontal cortex, an inactivation study was performed in a separate group of adult rats $(n=16)$. Infusions were given by removing obturators and inserting injection cannulae (28 gauge; Plastics One) that extended $1 \mathrm{~mm}$ beyond the guide cannulae. The injectors were connected via tubing to Hamilton syringes (Reno, NV) controlled by a syringe pump. Infusions were given in a volume of $0.3 \mu \mathrm{l}$ over $1 \mathrm{~min}$, and injectors were left in the cannulae for an additional $1 \mathrm{~min}$ to allow for drug diffusion. Baclofen and muscimol (bac/mus) were infused at a dose of
0.3/0.03 nmol/side, which are doses known to reversibly inactivate discrete brain regions (McFarland and Kalivas, 2001; Torregrossa et al, 2013). Control infusions were performed (order counterbalanced across all rats) where rats received the same volume of saline.

\section{Short-Term Spatial and Object Memory Tests}

Short-term memory was assessed after 3 weeks long-access WIN SA (Figure 3a). A subset of rats with a history of experimenter-administered WIN in adolescence $(n=9$ vehicle, 8 WIN) were tested immediately after exposure on PD52, and the remaining IP rats $(n=8$ vehicle, 8 WIN) were tested after a period of drug-free recovery on PD106 (Figure 5a). Rats were placed in an open field $(43 \mathrm{~cm} \times 43 \mathrm{~cm}$; Med Associates) under dim light and allowed to explore for $5 \mathrm{~min}$. After 2-3 days of habituation, rats were tested for novel object and spatial memory. In the object memory test, rats were allowed to explore two objects placed evenly from opposing corners. All objects were similar in dimension 
$(\sim 11 \mathrm{~cm} \times 11 \mathrm{~cm})$, and material (glass/ceramic) but had distinct textures and color patterns. Rats were returned to their homecages for a 35 min delay, then were returned to the open field for $5 \mathrm{~min}$ where one of the original objects was replaced with a novel object. Twenty-four hours after the object memory test, spatial memory was tested. Rats were allowed to explore two objects placed evenly from opposing corners, and after a $\sim 35$ min delay, were returned to the open field for $5 \mathrm{~min}$ where one of the original objects was now in a new spatial location. The testing arena and objects were thoroughly cleaned with $70 \%$ ethanol between testing. Data were collected and analyzed offline using AnyMaze software (Stoelting, Wood Dale, IL).

\section{Western Blot Analysis}

Short-access SA rats were killed by rapid decapitation, brains flash-frozen in isopentane on dry ice, and stored at $-80^{\circ} \mathrm{C}$. Prelimbic (PL) and infralimbic (IL) PFC were excised from $\sim 1 \mathrm{~mm}$-thick sections, and were fractionated into membrane- and non-membrane-bound components based on published protocols (Bañuelos et al, 2014). Protein concentrations were determined using the bicinchoninic acid assay (BCA Protein Assay; Thermo-Scientific Pierce, Waltham, MA), according to the manufacturer's protocol. Samples were denatured and reduced in sample buffer $(60 \mathrm{mM}$ Tris (pH 6.8), 10\% glycerol, $2 \%$ SDS, $0.1 \%$ bromphenol blue, and $5 \% 2-\beta$-mercaptoethanol), and heated to $90{ }^{\circ} \mathrm{C}$ for $5 \mathrm{~min}$.

Membrane and soluble fractions $(20 \mu \mathrm{g}$ total protein) were resolved by SDS-PAGE (4-12\% Tris-glycine gels; Invitrogen) and electrophoretically transferred to nitrocellulose membranes. Membranes were incubated in blocking solution (5\% non-fat dry milk in PBS containing $0.1 \%$ Tween 20) for 1 -h at room temperature. Membranes were incubated at $4{ }^{\circ} \mathrm{C}$ overnight with specific primary antibodies against the following proteins: $G A B A$ receptor subunits $G_{A B A}$ receptor subunit R2 ( $\left.\mathrm{GABA}_{\mathrm{B}} \mathrm{R} 2\right)(1: 1000$; Cell Signaling, Danvers, $\mathrm{MA})$ and $\mathrm{GABA}_{\mathrm{A}} \mathrm{R} 1 \alpha(1: 10$ 000; Abcam, Cambridge, UK); GAT-1 (1 : 5000; Abcam); GAD67 (1: 7000; EMD Millipore, Billerica, MA); GAD65 (1:1000 EMD Millipore); NMDA receptor subunit NR2B (1:500; EMD Millipore) and phosphorylated (serine 1303) pNR2B (1:500; EMD Millipore); and loading control GAPDH (1:1000; EMD Millipore). Antigen binding was visualized with secondary fluorescent antibodies (IRDye $800 \mathrm{CW}$ anti-rabbit, 1:5000; IRDye $680 \mathrm{CW}$ anti-mouse, 1:5000). All primary and secondary antibodies were diluted in blocking solution (50\% LI-COR Odyssey blocking buffer/50\% PBS). Protein expression was quantified using LI-COR-Odyssey imaging software, with each sample normalized to its own GAPDH expression, and expression for the WIN group within a gel normalized to average levels of the sucrose group.

\section{Statistical Analyses}

Differences in working memory accuracy were determined using a two-factor repeated measures ANOVA (group $\times$ delay). Latencies to complete correct or incorrect trials were analyzed by independent samples $t$-tests. Cue-induced reinstatement and incubation of craving in long-access SA groups was analyzed with one-way ANOVAs on active lever presses and inactive lever presses separately, followed by Bonferonni's-corrected $t$-tests. Protein expression in PL and IL cortices were analyzed by independent samples $t$-tests. Differences in short-term spatial and object memory were determined using two-factor ANOVAs (treatment $\times$ zone) separately for each treatment type (SA vs IP) and each test (spatial $v s$ object memory). All statistical analyses were performed using the SPSS software package version 21.0 (SPSS, Chicago, IL).

\section{RESULTS}

\section{Effects of Short-Access Cannabinoid Self-Administration during Adolescence on Working Memory}

In the first experiment, we examined whether adolescent short-access SA of the synthetic cannabinoid receptor agonist WIN55,212-2 (WIN) would alter working memory performance in adulthood (Figure 1a). Rats learned to self-administer sucrose pellets and WIN over a similar timeframe (Figure $1 \mathrm{~b}$ and $\mathrm{c}$ ). Importantly, rats responding for WIN pressed the active lever significantly more than the inactive lever (main effect of lever, $\mathrm{F}_{(11,242)}=9.00, p<0.001$ ).

Following $>35$ days of abstinence, rats remained drug free and were trained on a delay-match-to-sample working memory task as adults. There were no differences between rats with a history of adolescent sucrose or WIN SA during training on $0.5-12 \mathrm{~s}$ delays, as two-way analysis of variance with repeated measures ( $\mathrm{rmANOVA}$ ) indicated no effect of SA condition $\left(\mathrm{F}_{(6,21)}=0.15, p>0.05\right)$ or $\mathrm{SA} \times$ delay interaction $\left(\mathrm{F}_{(6,126)}=1.03, p>0.05\right)$ (Figure 1d). However, when delays increased to a range from $0-24 \mathrm{~s}, \mathrm{rmANOVA}$ revealed a significant main effect of delay $\left(\mathrm{F}_{(6,66)}=43.5, p<0.001\right)$, and main effect of SA $\left(F_{(1,11)}=11.88, p=0.006\right)$ (Figure 1e),

Figure 2 Short-access cannabinoid self-administration during adolescence alters prefrontal GABAergic and glutamatergic signaling. (a) GABA $\mathrm{B}$ receptor subunit GABA ${ }_{B}$ R2, (b) GAT-I, (c) phosphorylated NMDA receptor subunit NR2B on serine I 303 (pNR2B), and (d) GAD65 protein expression (protein of interest immunoreactivity relative to GAPDH immunoreactivity in the same sample), in prelimbic and infralimbic samples from rats with a history of adolescent sucrose or WIN55,2 I2-2 self-administration ( $n=6-8$ per group per protein). Ratios of the protein of interest/GAPDH immunoreactivity for rats that selfadministered WIN are expressed relative to the same ratio for rats that self-administered sucrose included on the same membrane. Representative western blots of the prelimbic and infralimbic regions of a rat from the sucrose SA group and a rat of the WIN SA group are shown above. Working memory performance across 8,12 , and $16 \mathrm{~s}$ delays was averaged to create a Mid Delay Performance score. GABA ${ }_{B} R 2$ expression in the (e) prelimbic region of the prefrontal cortex tended to relate to Mid Delay Performance, in that better performance corresponded to increased GABA $A_{B} R 2$ expression. (f) $G A B A_{B} R 2$ expression in the infralimbic did not correspond to better performance. (g) Implantation of bilateral guide cannulae aimed at the prelimbic prefrontal cortex and bilateral vehicle (saline) infusions did not affect working memory performance. Bilateral baclofen/muscimol infusions significantly reduced performance across all delays, particularly at high cognitive load $(\geqslant 4 \mathrm{~s})$. Values show group means \pm SEM; individual data points are displayed as circles; pfc: prefrontal cortex; ***** $p<0.00$ I, *** $p<0.01$, $\$ p<0.05$ vs 0.5 s. (h) Histological representation of infusion sites for bac/mus experiment. 
indicating that adult rats with a history of adolescent WIN SA performed significantly better than did adult rats with a history of adolescent sucrose SA, particularly when switched to a task with increased cognitive load. In addition, we examined the latency to respond on correct and incorrect trials, and latency to begin a new trial after a correct or incorrect response. There were no significant differences between the WIN and sucrose SA groups on any of these

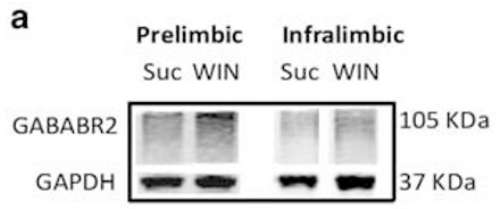

Relative Fold-Change of GABABR2

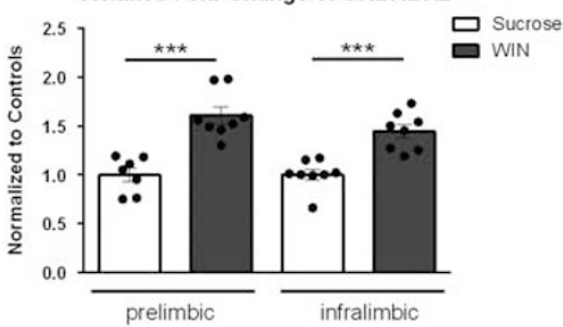

c
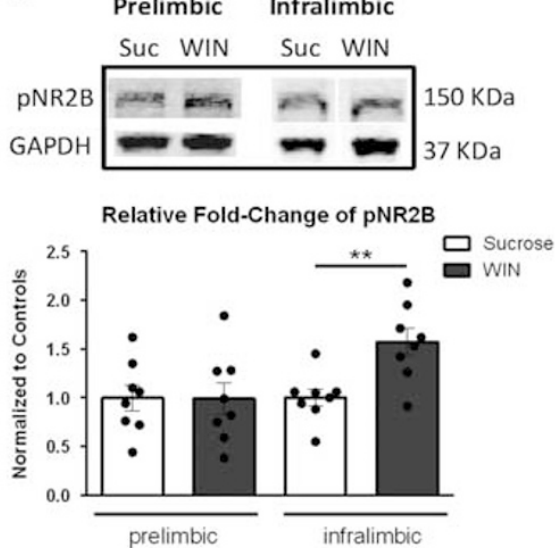

b
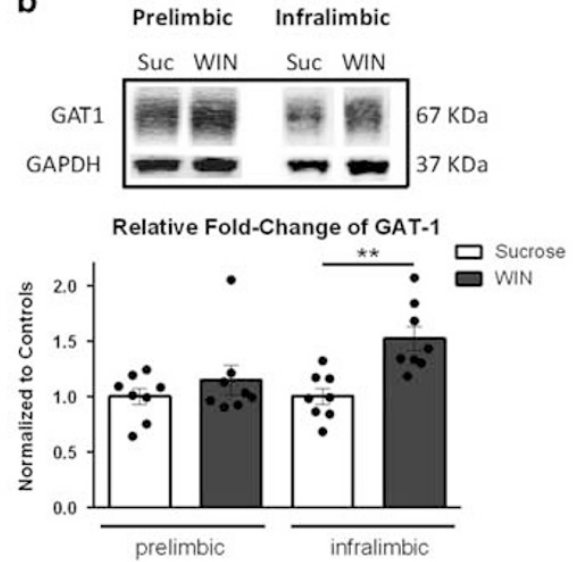

d
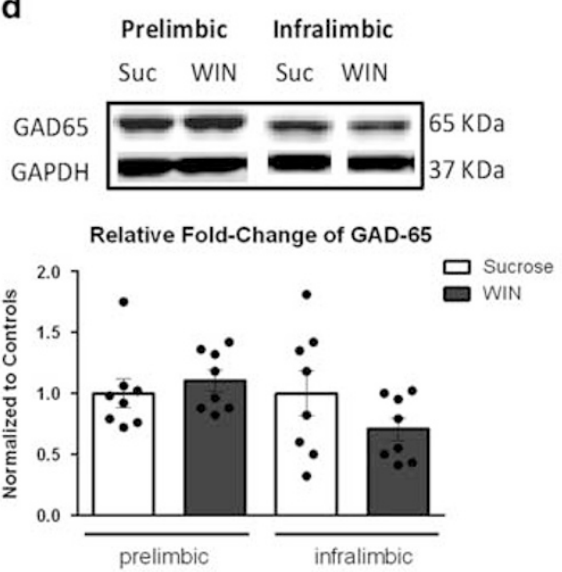
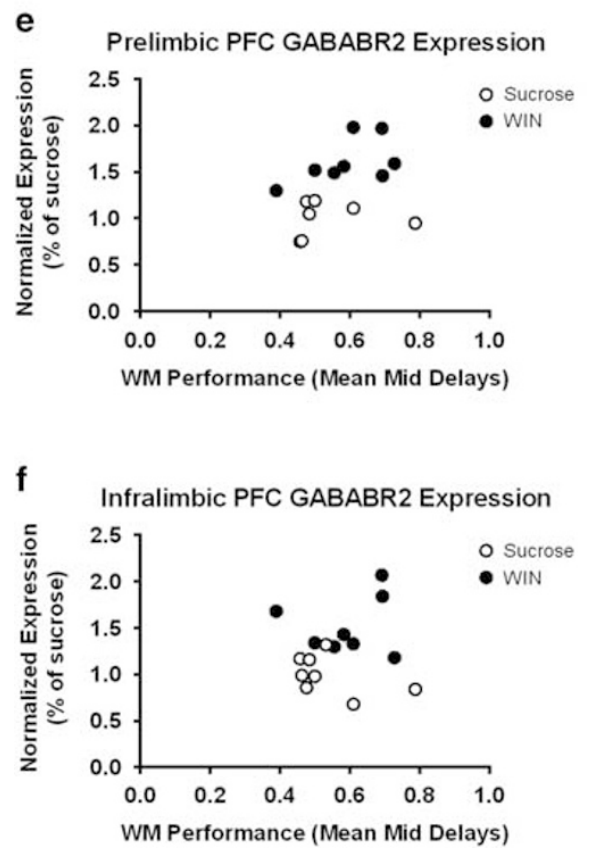

g

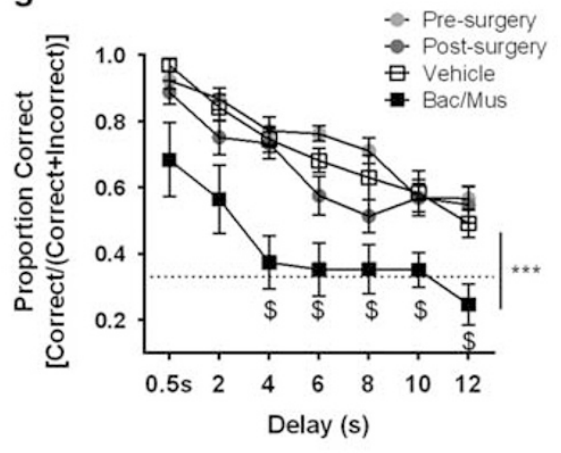

h

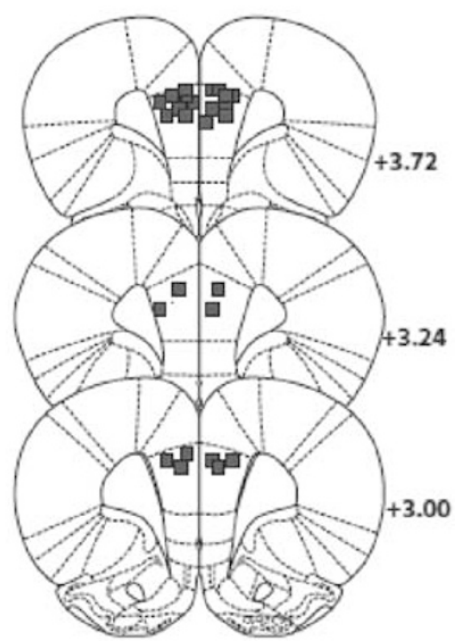


measures (all $p>0.05$; data not shown). However, there was a trend for the 2-h WIN SA group to respond faster on correct trials than the sucrose group $(p=0.08$; data not shown), suggesting that increased attention and/or motivation in this group may have contributed to their better performance.

\section{Effects of Adolescent Cannabinoid Self-Administration on Prefrontal Cortical Protein Expression}

We next examined changes in prefrontal GABAergic and glutamatergic-signaling protein expression in a subset of the adolescent sucrose and WIN SA groups $(n=6-8$ per group per protein). The proteins examined were chosen based on published research indicating involvement in working memory (Bañuelos et al, 2014). Rats with a history of WIN SA exhibited significantly increased expression of the $\mathrm{GABA}_{\mathrm{B}} \mathrm{R} 2$ in the PL $\left(\mathrm{t}_{(13)}=5.42, p<0.001\right)$, and IL PFC $\left(\mathrm{t}_{(14)}=5.09, p<0.001\right)$ (Figure 2a). No other proteins examined showed altered expression in the PL PFC. However, in the IL PFC, we observed increased expression of the GABA transporter (GAT-1) $\left(t_{(14)}=3.95, p=0.002\right.$; Figure $2 \mathrm{~b}$ ) and a phosphorylated subunit of the NMDA receptor on serine 1303, which is mediated by CaMKII and associated with membrane localization $(\mathrm{pNR} 2 \mathrm{~B})\left(\mathrm{t}_{(14)}=3.47\right.$, $p=0.004$; Figure 2c). Expression of the $\mathrm{GABA}_{\mathrm{A}} \mathrm{R} 1 \alpha$ subunit did not differ between groups ( $p>0.05$; data not shown). Expression of the GABA-synthesizing enzymes GAD65 and GAD67 did not differ between groups ( $p>0.05$; Figure $2 \mathrm{~d}$, and data not shown).

To relate changes in GABAergic signaling protein expression to prefrontal-dependent cognitive abilities, we correlated individual protein expression levels with performance on the working memory task. We averaged performance across delays where accuracy was greater than chance and that were presumed to produce high cognitive load (8, 12 , and $16 \mathrm{~s}$ ) to create an index of individual working memory ability (Mean Mid Delay). As shown in Figure 2e, expression of $\mathrm{GABA}_{\mathrm{B}} \mathrm{R} 2$ in the PL region seemed to relate to working memory ability, such that better performance corresponded to increased $\mathrm{GABA}_{\mathrm{B}} \mathrm{R} 2$ expression (Pearson's correlation between PL GABA ${ }_{B}$ R2 expression and Mean Mid Delay Performance: $r=0.38, p=0.16$ ). However, a larger sample size is needed to definitively confirm or reject this potential relationship. There was not a significant correlation between performance and $\mathrm{GABA}_{\mathrm{B}} \mathrm{R} 2$ expression in the IL region, however (Pearson's correlation between IL GABA ${ }_{B} R 2$ expression and Mean Mid Delay Performance: $r=0.10$, $p=0.72$; Figure $2 \mathrm{f}$ ). Working memory performance was not significantly correlated with any of the other proteins examined.

We confirmed that accurate performance on the working memory task required activity in the PL PFC in a separate cohort of adults, as intra-PL infusion of the $\mathrm{GABA}_{\mathrm{A} / \mathrm{B}}$ receptor agonists baclofen/muscimol $(0.3 \mathrm{nmol} / 0.03 \mathrm{nmol})$ significantly reduced accuracy across delays with high cognitive load (main effect of treatment: $F_{(3,48)}=20.53$, $p<0.001$; Figure $2 \mathrm{~g}$ ). We cannot rule out a potential contribution of activity in the IL cortex to working memory, as it is possible the infusion spread to this region. However, Figure $2 \mathrm{~h}$ illustrates that the location of placements in the PL cortex were quite dorsal to the IL cortex ( $n=14$; two misses were excluded from analyses).

\section{Effects of Long-Access Cannabinoid Self-Administration during Adolescence on Short-Term Memory and Working Memory}

In the second experiment, we examined whether extendedaccess SA of WIN during adolescence would result in greater WIN exposure, and subsequently impair working memory in adulthood (Figure 3a). Figure 3b illustrates the SA acquisition curve for WIN in long-access (6-h) sessions. Rats were trained as in experiment 1, but sessions increased from 1 to 6-h on the fifth day of training. Rats responding for WIN pressed the active lever significantly more than the inactive lever (main effect of lever, $\mathrm{F}_{(1,11)}=115.60, p<0.001$ ). Active lever presses were stable by day 12 of SA (main effect of day, $\mathrm{F}_{(18,198)}=8.29, p<0.001$; day $\times$ lever interaction, $\left.\mathrm{F}_{(18,198)}=4.19, p<0.001\right)$. However, rats did not demonstrate any evidence of 'escalation of intake' with 6-h access as has been reported for adult rats responding for multiple other drugs of abuse (Edwards and Koob, 2013), and did not substantially differ in their total intake from the 2-h access group. We examined the pattern of intake across the 6-h session, and it was evenly distributed across the first and second halves of the session (no significant difference in average intake during first $3-\mathrm{h}$ and second 3-h: $\mathrm{t}_{(14)}=0.95$, $p=0.36)$.

Twenty-four hours after the final WIN exposure, spatial recognition and object recognition were assessed, to measure acute effects of WIN SA on short-term memory, using tasks previously reported to be sensitive to experimenter administered WIN (Abush and Akirav, 2012; O'Shea et al, 2004, 2006; Schneider et al, 2008). In the spatial recognition test, rats with a history of sucrose SA demonstrated intact shortterm spatial memory, with increased investigation of an object in a novel spatial location (Figure 3c). The WIN SA group behaved similarly, with no evidence of a memory deficit (Figure 3c) (two-way ANOVA, main effect of zone: $\mathrm{F}_{(1,44)}=62.6, \quad p<0.001$; no main effect of treatment: $\mathrm{F}_{(1,44)}=1.54, \quad p>0.05$; no treatment $\times$ zone interaction: $\left.\mathrm{F}_{(1,44)}=0.97, p>0.05\right)$. Contrary to what is typically observed in the object recognition test (Antunes and Biala, 2012; O'Shea et al, 2006; Schneider and Koch, 2003), all rats tended to avoid rather than prefer exploring a novel object. This may be due to the age of testing, or another unknown parameter. Nevertheless, avoidance still indicates the ability to discriminate between novel and familiar objects. As such, rats with a history of sucrose SA showed intact short-term object memory, as did rats with a history of WIN SA (Figure 3d) (main effect of zone: $\mathrm{F}_{(1,44)}=16.93, p=0.002$; no main effect of treatment: $\mathrm{F}_{(1,44)}=0.87, p>0.05$; no treatment $\times$ zone interaction: $\left.\mathrm{F}_{(1,44)}=0.86, p>0.05\right)$. Together, these results suggest that adolescent WIN SA does not impair short-term memory under these experimental conditions.

Rats were then trained on the working memory task, as in experiment 1 (11 day drug washout period prior to any training). Similar to short-access SA, there were no differences between rats with a history of adolescent sucrose or WIN SA at any point in task training or when delays were expanded to $0.5-12 \mathrm{~s}$ ( $p>0.05$; data not shown). However, when the delay range was expanded to $0.5-24 \mathrm{~s}$ (Figure $3 \mathrm{e}$ ), 
a

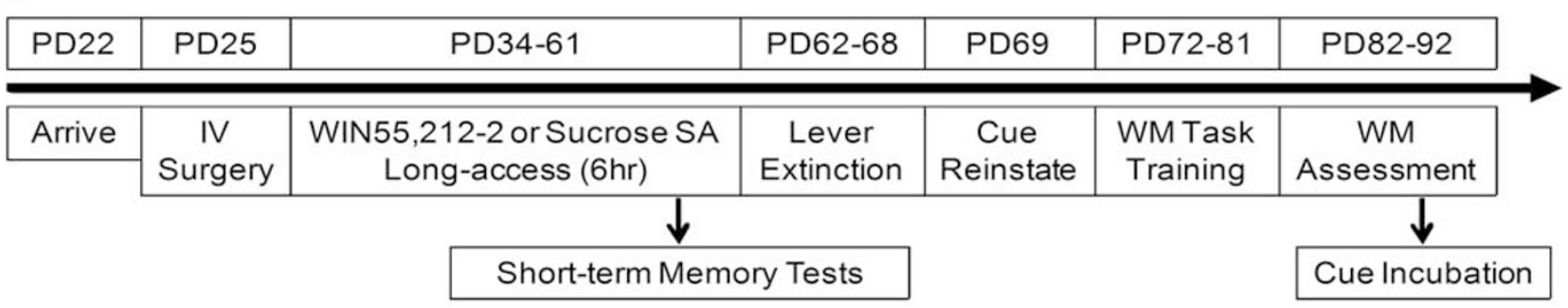
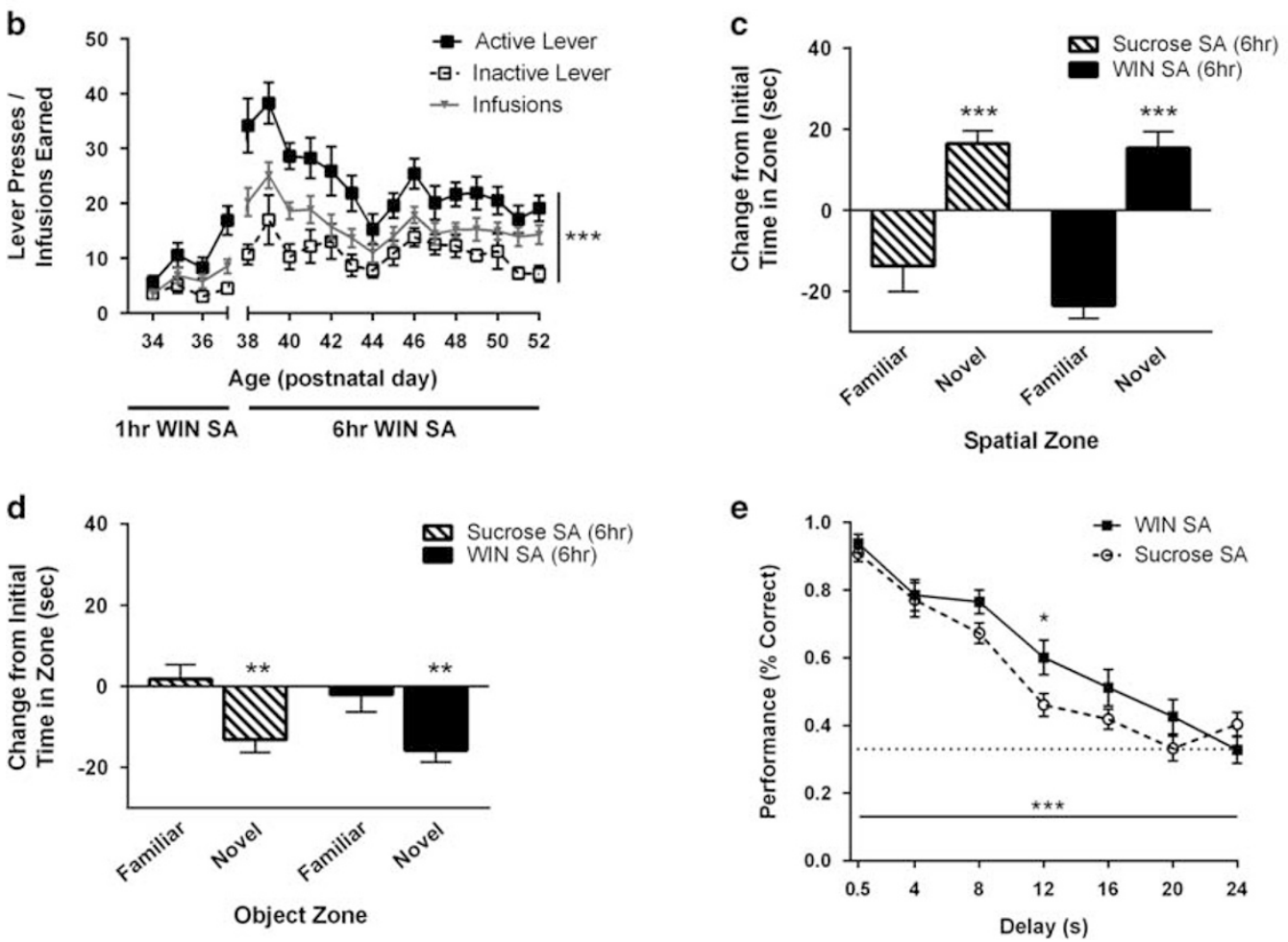

Figure 3 Long-access cannabinoid self-administration during adolescence has no effect on short-term memory, but improves working memory performance, in adulthood. (a) Experimental timeline. Rats ( $n=12$ per group) were trained to self-administer sucrose pellets or WIN55,2I2-2 (0.0 I25 mg/kg/ infusion). (b) Active lever presses for WIN were significantly higher than inactive presses across training days. Infusions earned are shown in gray. Twenty-four hours after last self-administration session, rats were tested for short-term spatial (c) and object (d) memory. Rats with a history of sucrose or WIN SA during adolescence exhibited intact short-term spatial memory, with increased time spent investigating an object in a novel location. Both sucrose SA and WIN SA rats discriminated familiar from novel objects, and thus exhibited intact short-term memory for objects as well. (e) Rats trained to self-administer WIN in 6-h sessions during adolescence showed improved working memory performance in adulthood with an 0-24s delay range. The proportion of correct responses for WIN SA rats was greater than sucrose SA controls across delays with high cognitive load. Values show group means \pm SEM; $* * * * 2<0.00$ I, *** $p<0.0$ I, $* p<0.05$.

we again observed better working memory performance in the WIN SA group, with rmANOVA revealing a significant main effect of delay $\left(\mathrm{F}_{(6,132)}=80.5, p=<0.001\right)$, and group $\times$ delay interaction $\left(\mathrm{F}_{(6,132)}=2.25, p=0.04\right)$. Post hoc analyses revealed that WIN SA rats performed significantly better at delays with high cognitive load (12s).

\section{Cue-Induced Reinstatement of WIN Seeking}

We also examined whether long-access SA was able to elicit relapse-like behavior or the incubation-of-craving phenomenon that has been established with other drugs of abuse (Grimm et al, 2001; Pickens et al, 2011). Figure 4a shows lever extinction training data across days. The following day (8th day of abstinence), rats were tested for cue-induced reinstatement of WIN seeking in a 30-min session. Rats with a history of long-access WIN SA significantly increased the rate of responding on the active lever compared with the last day of extinction (paired $t$-test, rate of lever presses on last day of extinction $v s$ rate of lever presses on reinstatement: $\mathrm{t}_{(11)}=3.08, p=0.010$; Figure $\left.4 \mathrm{~b}\right)$. Rats were tested again 3 weeks later (29th day of abstinence), and the rate of responding on the formerly active lever was further increased, indicating an incubation of craving (paired $t$-test, rate of lever presses on reinstatement day $v s$ on incubation day: $t_{(11)}=5.59, p<0.001$; Figure $\left.4 b\right)$. The total number of lever presses are also shown (Figure 4c), which also indicates a significant increase in responding from abstinence day 8-29 $(p<0.001)$; and the lack of difference between the last day of extinction and day 8 of abstinence (reinstatement) is due to the difference in session length. 
a

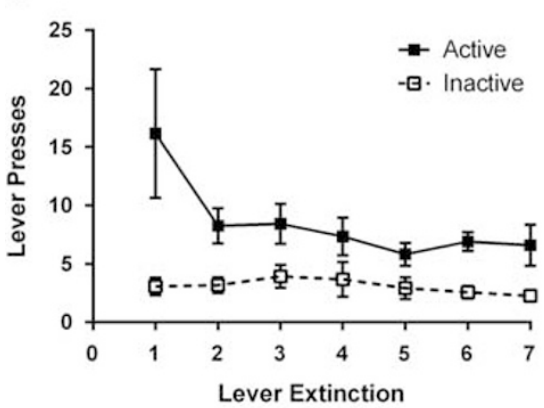

b

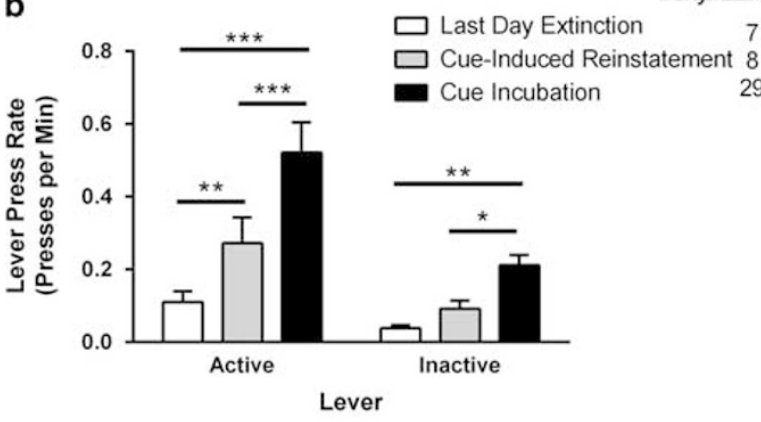

C

Days Abstinent

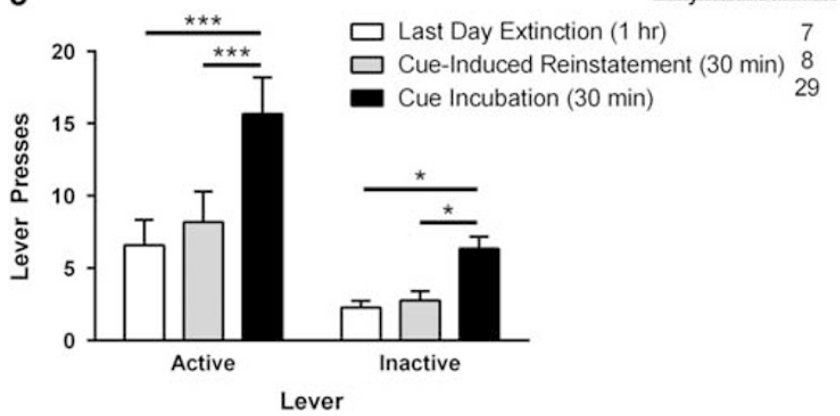

Figure 4 Rats with a history of long-access WIN55,2 I2-2 self-administration during adolescence demonstrate cue-induced reinstatement of drug-seeking. (a) WIN SA rats were extinguished on response-contingent drug administration during I-h sessions for I week. Active lever presses reduced to similar numbers as inactive lever presses. (b) Relapse of drug-seeking behavior was tested I and $2 \mathrm{I}$ days later (corresponding to 8 and 29 days of abstinence). Rats significantly increased responding on day 8 , indicating reinstatement of drug-seeking behavior; and this effect was magnified when rats were tested on day 29 of abstinence, indicating an incubation of craving. (c) Total number of lever presses for the last day of extinction (abstinence day 7; I-h session), day 8 of abstinence (30-min session) and day 29 of abstinence (30-min session). Rats had a significant increase in responding from abstinence day 8-29, and the lack of difference between the last day of extinction and day 8 of abstinence is due to the difference in session length. Values show group means \pm SEM; $* * * *<<0.00$ I, *** $p<0.01$, $*$ $p<0.05$.

\section{Immediate and Prolonged Effects of Experimenter- Administered Cannabinoids During Adolescence on Cognition}

Experimenter-administration of WIN has been reported to cause short-term memory deficits (Abush and Akirav, 2012; O'Shea et al, 2006), which we did not observe after SA. In order to determine whether differences in dose received explain our conflicting findings, we first verified that we could replicate previous studies showing that $1.2 \mathrm{mg} / \mathrm{kg} / \mathrm{day}$ of IP WIN during adolescence produces short-term memory deficits. We then determined whether that dosing regimen would affect adult working memory performance (Figure 5a).

Rats were tested for short-term spatial and object memory $24 \mathrm{~h}$ after the last IP WIN injection. As with the SA groups, the IP vehicle group exhibited intact short-term spatial memory (Figure 5b). However, rats with a history of IP WIN had impaired short-term spatial memory (Figure 5b) (twoway ANOVA, main effect of zone: $\mathrm{F}_{(1,30)}=7.05, p=0.013$; main effect of treatment: $\mathrm{F}_{(1,30)}=5.72, p=0.023$; treatment $\times$ zone interaction: $\left.\mathrm{F}_{(1,30)}=6.58, p=0.02\right)$. Similar to the SA experiment, rats with a history of IP vehicle tended to avoid the novel object, while rats with a history of IP WIN failed to discriminate between the familiar and novel objects (Figure $5 c$ ) (no main effect of zone: $\mathrm{F}_{(1,30)}=2.42, p>0.05$; no main effect of treatment: $F_{(1,30)}=0.47, p>0.05$; trend for zone $\times$ treatment interaction: $\left.\mathrm{F}_{(1,30)}=3.43, p>0.07\right)$. This suggests that IP WIN during adolescence impaired shortterm object memory as well.

Next, these rats were trained on the working memory task (17 day drug washout period prior to any training). Similar to short- and long-access SA, there were no differences between rats with a history of IP vehicle or IP WIN during adolescence at any point in task training $(p>0.05$; data not shown). When the delay range was expanded to 0-24 s (Figure 5d), rmANOVA revealed a significant main effect of delay $\left(\mathrm{F}_{(6,186)}=88.0, p=<0.001\right)$, but no effect of IP treatment $\left(\mathrm{F}_{(1,31)}=0.45, p>0.05\right)$, or treatment $\times$ delay interaction $\left(\mathrm{F}_{(6,186)}=0.91, p>0.05\right)$, indicating that IP WIN in adolescence did not alter working memory function. However, a slight reduction in accuracy at the $8 \mathrm{~s}$ delay raises the possibility that exposure to even higher doses of WIN may have detrimental consequences for working memory.

Finally, because working memory performance was not significantly impaired, we examined whether short-term memory function remained impaired after prolonged abstinence. A separate cohort of rats that had received IP WIN or vehicle during adolescence were tested in the shortterm spatial memory task, as described, with the exception that the test occurred 52 days after final WIN exposure (Figure 5a). A history of IP vehicle resulted in intact shortterm spatial memory, as expected (Figure 5e). Likewise, rats 
a

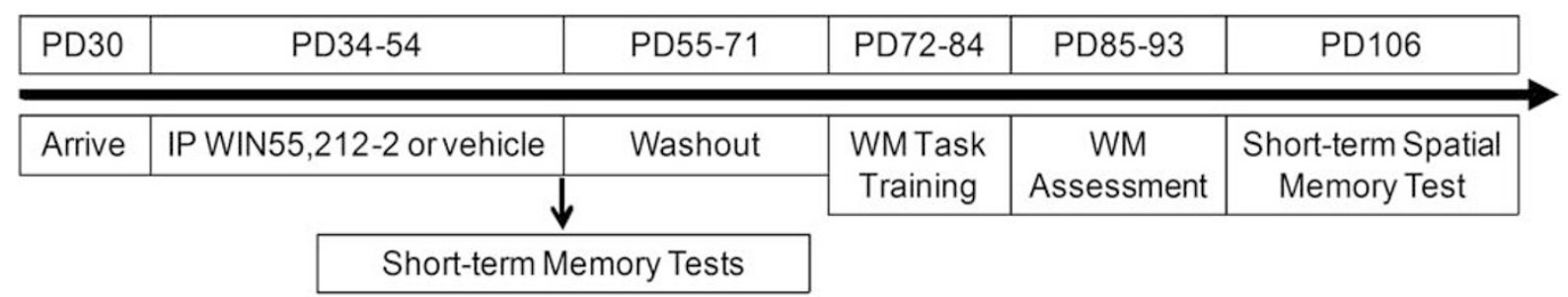

b
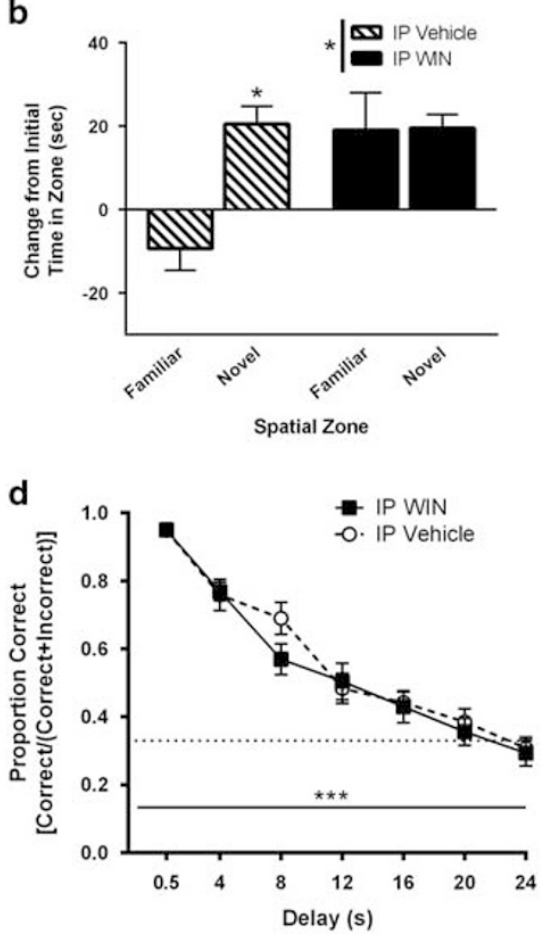

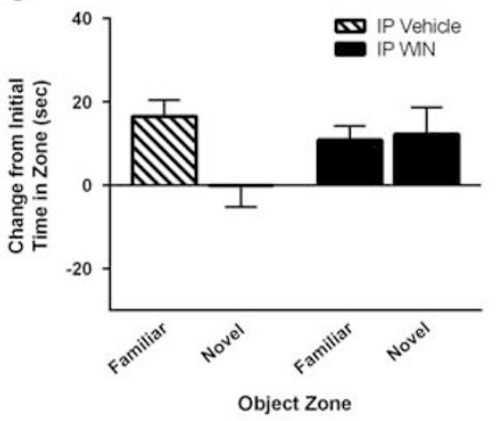

e

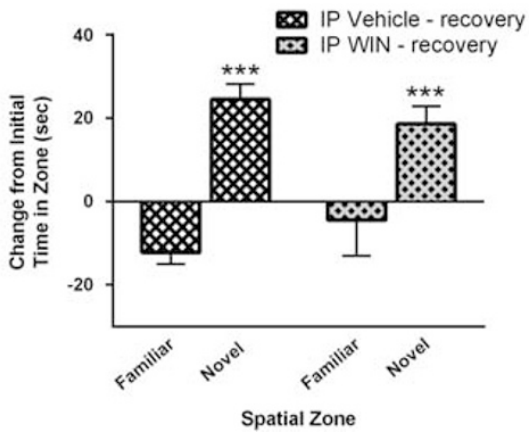

Figure 5 Experimenter-administered cannabinoids during adolescence acutely impair short-term memory, but have no effect on working memory performance in adulthood. (a) Experimental timeline. Rats received daily intraperitoneal (IP) injections of WIN $(I .2 \mathrm{mg} / \mathrm{kg} ; n=16)$ or vehicle $(n=\mid 7)$ during adolescence. (b) Twenty-four hours after the last IP injection, short-term spatial (b) and object (c) memory was assessed in one cohort of IP WIN ( $n=8$ ) and IP vehicle $(n=9)$ rats. Rats with a history of IP vehicle exhibited intact short-term spatial memory, whereas rats with a history of IP WIN during adolescence did not discriminate between the two locations, indicating a short-term spatial memory deficit. Rats with a history of IP vehicle during adolescence tended to discriminate familiar from novel objects, and thus exhibited intact short-term memory for objects. Rats with a history of IP WIN during adolescence did not discriminate between the two objects, indicating a deficit in short-term recognition memory. (d) Adult working memory was not different in rats that received IP WIN or IP vehicle during adolescence. (e) After extended abstinence from IP WIN $(n=8)$ or IP vehicle $(n=8)$, both groups exhibited intact short-term memory, indicating recovery from the acute deficits induced by WIN. Values show group means \pm SEM; $* * * *<<0.00 I, * p<0.05$.

with a history of IP WIN did not have deficits in short-term memory (Figure 5e) (two-way ANOVA, main effect of zone: $\mathrm{F}_{(1,28)}=31.5, \quad p<0.001$; no main effect of treatment: $\mathrm{F}_{(1,28)}=0.03, \quad p>0.05 ;$ no treatment $\times$ zone interaction: $\left.\mathrm{F}_{(1,28)}=1.65, p>0.05\right)$, suggesting that the acute deficits described above were temporary, and that memory recovered with continued abstinence.

Thus, we were able to replicate prior reports showing that $1.2 \mathrm{mg} / \mathrm{kg} /$ day WIN can produce acute deficits in short-term memory that were not observed after SA of WIN. However, the average daily dose of self-administered WIN was $0.216 \mathrm{mg} / \mathrm{kg}$, and the highest dose any rat self-administered during a session was $0.6 \mathrm{mg} / \mathrm{kg}$, suggesting that memory impairing doses of a cannabinoid receptor agonist are not readily achieved under SA conditions.

\section{DISCUSSION}

The aim of the present study was to determine whether an adolescent cannabinoid SA model could be developed to explore the abuse liability of adolescent-onset cannabinoid use and for determining long-term effects of cannabinoid exposure under SA conditions on cognitive function. Contrary to expectations, adolescent SA of WIN improved performance on a delay-match-to-sample working memory task in adulthood. The same results were observed in two cohorts of rats, regardless of whether they received 2- or 6-h access SA sessions. Moreover, we found no evidence of either acute or long-lasting deficits in short-term memory for a novel object or location, further suggesting that cannabinoid exposure under SA conditions is less detrimental than previous studies using experimenter-administration would suggest. 


\section{Conditions of Cannabinoid Exposure in Adolescence Differentially Impact Cognition}

The difference between SA and experimenter-administration could be owing to having volitional control over intake, differences in the pattern of intake with the accumulation of small IV doses of WIN, or differences in the total dose of WIN achieved. Indeed, rats in our study self-administered a maximum of $0.6 \mathrm{mg} / \mathrm{kg}$, and averaged $\sim 0.22 \mathrm{mg} / \mathrm{kg} /$ day. In comparison, studies using experimenter-administered dosing in rodents only find short-term memory deficits at daily doses of $1.2 \mathrm{mg} / \mathrm{kg}$, which we replicated here (Abush and Akirav, 2012). In addition, adolescent primates receiving repeated experimenter-administered THC were impaired in a spatial-based, but not object-based, memory task, in a dosedependent manner (Verrico et al, 2012). Therefore, differences in dose alone may be sufficient to explain our results. Indeed, experimenter-administration of $1.2 \mathrm{mg} / \mathrm{kg} /$ day WIN did not produce working memory improvements, however, it also did not produce deficits, suggesting that the lower dose exposure and/or control over intake that occurred under SA conditions was necessary for working memory improvements. It is also important to note that a significant abstinence period was introduced between WIN exposure and working memory testing; thus, it is possible that temporary working memory impairments could have been observed, were we able to test working memory immediately following WIN exposure. Indeed, Lewis and colleagues have reported a blunted trajectory of spatial working memory improvements in adolescent primates receiving experimenter-administered THC and tested 24-72 h after exposure (Verrico et al, 2014). Finally, the rewarding/ aversive properties of WIN may have had a role in the experimental differences observed. Self-administration of WIN indicated a reinforcing effect (rats continued to respond for cues previously paired with drug delivery), whereas experimenter-administration of WIN may be a pharmacological stressor (Quinn et al, 2008). The anxiogenic stress-like effects of forced exposure may be the cause of cognitive deficits we observed acutely in the short-term memory tests, as well as in those reported in previous studies (Klein et al, 2011; O'Shea et al, 2004; Quinn et al, 2008).

\section{Altered Prefrontal GABAergic Tone May Mediate Changes in Cognition Observed After Adolescent WIN Self-Administration}

In addition, we were able to identify changes in protein expression in the PFC that may mediate the improvement in working memory produced by adolescent WIN SA. Interestingly, although we found that activity of the PL PFC was required for accurate working memory performance in our task as reported by others (Gilmartin et al, 2013; Horst and Laubach, 2009), we observed the majority of changes in protein expression in the IL PFC. In both the PL and IL we found increased surface expression of subunit 2 of the $\mathrm{GABA}_{\mathrm{B}} \mathrm{R}$ in the WIN SA group, however, only PL GABA $\mathrm{B} 2$ expression tended to be positively associated with performance. Clinical studies in healthy subjects using combined TMS-EEG over the dorsolateral PFC also find that greater $\mathrm{GABA}_{\mathrm{B}} \mathrm{R}$-mediated inhibition is associated with better working memory performance (Daskalakis et al, 2008; Rogasch et al, 2015).

Interestingly, age-related declines in working memory are also associated with changes in prefrontal (combined PL and IL) expression of the $\mathrm{GABA}_{\mathrm{B}} \mathrm{R}$ subunit 2, GAT-1, GAD65, and GAD67 (Bañuelos et al, 2014). The expression pattern of the WIN SA group appears more like the young rats, consistent with better working memory performance. Bañuelos et al also found that $\mathrm{GABA}_{\mathrm{B}} \mathrm{R}$ inhibition selectively impaired working memory in young rats, suggesting that different mechanisms may mediate working memory impairments associated with aging. Indeed, previous work testing the effects of GABA receptor manipulations on working memory suggest that there is likely a tight inverted$\mathrm{U}$ function, where too much or too little GABA signaling can produce working memory deficits (Auger and Floresco, 2015; Escher and Mittleman, 2004).

\section{Adolescent WIN Self-Administration Induces Some Addiction-Like Behaviors}

Despite the lack of working memory deficits, we observed clear indications of addiction-like effects from WIN SA, including cue-induced reinstatement and incubation of craving. However, extended access (6-h) SA conditions did not lead to escalation of intake over days, as has been reported for other drugs of abuse (Edwards and Koob, 2013). The consistent amount of intake observed, regardless of session length, suggests that the mechanisms mediating escalation for other drugs of abuse are not applicable to cannabinoids, or that there are delayed aversive effects that limit further SA. Indeed, higher unit doses of WIN are not readily self-administered by rats (Fattore et al, 2001). Nevertheless, escalation of intake has not been reported in adolescents to our knowledge, which raises the possibility that age may also influence this phenomenon.

\section{CONCLUSIONS}

In conclusion, we report the first rodent model of adolescent cannabinoid SA, and have found that adolescent cannabinoid SA alone is not sufficient to produce long-lasting memory deficits. Our data seem consistent with the human literature, which indicates that the largest memory deficits in cannabis users are observed in those who maintain high levels of intake into adulthood, and not in those individuals who use infrequently or are not currently using (Crean et al, 2011a,b; Ganzer et al, 2016; Solowij and Battisti, 2008). Finally, our data do support that cannabinoid SA can result in relapse-like behavior, underscoring the potential abuse liability of cannabinoids in adolescents.

\section{FUNDING AND DISCLOSURE}

The authors declare no conflict of interest.

\section{ACKNOWLEDGMENTS}

We would like to acknowledge technical aid from Megan Bertholomey, Jenna Parrish, and Matthew Rich, and the NIH 
drug supply program. This work was supported by NIH grant DA031745 and the Pennsylvania Department of Health.

\section{REFERENCES}

Abush H, Akirav I (2012). Short- and long-term cognitive effects of chronic cannabinoids administration in late-adolescence rats. PLoS One 7: e31731.

Antunes M, Biala G (2012). The novel object recognition memory: neurobiology, test procedure, and its modifications. Cogn Process 13: 93-110.

Auger ML, Floresco SB (2015). Prefrontal cortical GABA modulation of spatial reference and working memory. Int J Neuropsychopharmacol 18: 1-11.

Bañuelos C, Beas BS, McQuail JA, Gilbert RJ, Frazier CJ, Setlow B et al (2014). Prefrontal cortical GABAergic dysfunction contributes to age-related working memory impairment. $J$ Neurosci 34: 3457-3466.

Becker MP, Collins PF, Luciana M (2014). Neurocognition in college-aged daily marijuana users. J Clin Exp Neuropsychol 36: 379-398.

Buchy L, Seidman LJ, Cadenhead KS, Cannon TD, Cornblatt BA, McGlashan TH et al (2015). Evaluating the relationship between cannabis use and IQ in youth and young adults at clinical high risk of psychosis. Psychiatry Res 230: 878-884.

Cohen Kadosh K, Krause B, King AJ, Near J, Cohen Kadosh R (2015). Linking GABA and glutamate levels to cognitive skill acquisition during development. Hum Brain Mapp 36: 4334-4345.

Compte A, Brunel N, Goldman-Rakic PS, Wang XJ (2000). Synaptic mechanisms and network dynamics underlying spatial working memory in a cortical network model. Cereb Cortex 10: 910-923.

Crean RD, Crane NA, Mason BJ (2011a). An evidence based review of acute and long-term effects of cannabis use on executive cognitive functions. J Addict Med 5: 1-8.

Crean RD, Tapert SF, Minassian A, Macdonald K, Crane NA, Mason BJ (2011b). Effects of chronic, heavy cannabis use on executive functions. J Addict Med 5: 9-15.

Daskalakis ZJ, Farzan F, Barr MS, Rusjan PM, Favalli G, Levinson AJ et al (2008). Evaluating the relationship between long interval cortical inhibition, working memory and gamma band activity in the dorsolateral prefrontal cortex. Clin EEG Neurosci 39: 150-155.

Edwards S, Koob GF (2013). Escalation of drug self-administration as a hallmark of persistent addiction liability. Behav Pharmacol 24: 356-362.

Escher T, Mittleman G (2004). Effects of ethanol and GABAB drugs on working memory in $\mathrm{C} 57 \mathrm{BL} / 6 \mathrm{~J}$ and DBA/2J mice. Psychopharmacology (Berl) 176: 167-175.

Fattore L, Cossu G, Martellotta CM, Fratta W (2001). Intravenous self-administration of the cannabinoid CB1 receptor agonist WIN 55,212-2 in rats. Psychopharmacology (Berl) 156: 410-416.

Gage SH, Matthew H, Zammit SG (2015). Association between cannabis and psychosis: epidemiological evidence. Biol Psychiatry 79: $549-556$.

Ganzer F, Bröning S, Kraft S, Sack P-M, Thomasius R (2016). Weighing the evidence: a systematic review on long-term neurocognitive effects of cannabis use in abstinent adolescents and adults. Neuropsychol Rev 26: 186-222.

Gilmartin MR, Miyawaki H, Helmstetter FJ, Diba K (2013). Prefrontal activity links nonoverlapping events in memory. J Neurosci 33: 10910-10914.

Grimm JW, Hope BT, Wise RA, Shaham Y (2001). Neuroadaptation. Incubation of cocaine craving after withdrawal. Nature 412: 141-142.

Gunderson EW, Haughey HM, Ait-Daoud N, Joshi AS, Hart CL (2012). 'Spice' and 'K2' herbal highs: a case series and systematic review of the clinical effects and biopsychosocial implications of synthetic cannabinoid use in humans. Am J Addict 21: 320-326.

Hooper SR, Woolley D, De Bellis MD (2014). Intellectual, neurocognitive, and academic achievement in abstinent adolescents with cannabis use disorder. Psychopharmacology (Berl) 231: 1467-1477.

Horst NK, Laubach M (2009). The role of rat dorsomedial prefrontal cortex in spatial working memory. Neuroscience 164: 444-456.

Jager G, Kahn RS, Van Den Brink W, Van Ree JM, Ramsey NF (2006). Long-term effects of frequent cannabis use on working memory and attention: an fMRI study. Psychopharmacology (Berl) 185: $358-368$.

Justinova Z, Mascia P, Wu H-Q, Secci ME, Redhi GH, Panlilio LV et al (2013). Reducing cannabinoid abuse and preventing relapse by enhancing endogenous brain levels of kynurenic acid. Nat Neurosci 16: 1652-1661.

Klein C, Karanges E, Spiro A, Wong A, Spencer J, Huynh T et al (2011). Cannabidiol potentiates $\Delta^{9}$-tetrahydrocannabinol (THC) behavioural effects and alters THC pharmacokinetics during acute and chronic treatment in adolescent rats. Psychopharmacology (Berl) 218: 443-457.

Lefever TW, Marusich JA, Antonazzo KR, Wiley JL (2014). Evaluation of WIN 55,212-2 self-administration in rats as a potential cannabinoid abuse liability model. Pharmacol Biochem Behav 118: 30-35.

Marconi A, Di Forti M, Lewis CM, Murray RM, Vassos E (2016). Meta-analysis of the association between the level of cannabis use and risk of psychosis. Schizophr Bull 42: 1262-1269.

McFarland K, Kalivas PW (2001). The circuitry mediating cocaineinduced reinstatement of drug-seeking behavior. J Neurosci 21: 8655-8663.

Meier MH, Caspi A, Ambler A, Harrington H, Houts R, Keefe RSE et al (2012). Persistent cannabis users show neuropsychological decline from childhood to midlife. Proc Natl Acad Sci USA 109: E2657-E2664.

Michels L, Martin E, Klaver P, Edden R, Zelaya F, Lythgoe DJ et al (2012). Frontal GABA levels change during working memory. PLoS One 7: e31933.

Miech RA, Johnston L, O’Malley PM, Bachman JG, Schulenberg J, Patrick ME (2015). Trends in use of marijuana and attitudes toward marijuana among youth before and after decriminalization: the case of California 2007-2013. Int J Drug Policy 26: 336-344.

Mokrysz C, Landy R, Gage S, Munafo M, Roiser J, Curran H (2016). Are IQ and educational outcomes in teenagers related to their cannabis use? A prospective cohort study. J Psychopharmacol 30: 159-168.

Moss HB, Chen CM, Yi H-Y (2014). Early adolescent patterns of alcohol, cigarettes, and marijuana polysubstance use and young adult substance use outcomes in a nationally representative sample. Drug Alcohol Depend 136: 51-62.

O’Shea M, McGregor IS, Mallet PE (2006). Repeated cannabinoid exposure during perinatal, adolescent or early adult ages produces similar longlasting deficits in object recognition and reduced social interaction in rats. J Psychopharmacol 20: 611-621.

O'Shea M, Singh ME, McGregor IS, Mallet PE (2004). Chronic cannabinoid exposure produces lasting memory impairment and increased anxiety in adolescent but not adult rats. J Psychopharmacol 18: 502-508.

Paxinos G, Watson C (2005). The Rat Brain in Stereotaxic Coordinate. Elsevier Academic Press.

Pickens CL, Airavaara M, Theberge F, Fanous S, Hope BT, Shaham Y (2011). Neurobiology of the incubation of drug craving. Trends Neurosci 34: 411-420.

Quinn HR, Matsumoto I, Callaghan PD, Long LE, Arnold JC, Gunasekaran N et al (2008). Adolescent rats find repeated Delta (9)-THC less aversive than adult rats but display greater residual 
cognitive deficits and changes in hippocampal protein expression following exposure. Neuropsychopharmacology 33: 1113-1126.

Renard J, Rushlow WJ, Laviolette SR (2016). What can rats tell us about adolescent cannabis exposure? Insights from preclnical research. Can J Psychiatry 61: 328-334.

Rogasch NC, Daskalakis ZJ, Fitzgerald PB (2015). Cortical inhibition of distinct mechanisms in the dorsolateral prefrontal cortex is related to working memory performance: a TMS-EEG study. Cortex 64: 68-77.

Rogeberg O (2013). Correlations between cannabis use and IQ change in the Dunedin cohort are consistent with confounding from socioeconomic status. Proc Natl Acad Sci USA 110: 4251-4254.

Schneider M, Koch M (2003). Chronic pubertal, but not adult chronic cannabinoid treatment impairs sensorimotor gating, recognition memory, and the performance in a progressive ratio task in adult rats. Neuropsychopharmacology 28: 1760-1769.

Schneider M, Schömig E, Leweke FM (2008). Acute and chronic cannabinoid treatment differentially affects recognition memory and social behavior in pubertal and adult rats. Addict Biol 13: 345-357.

Solowij N, Battisti R (2008). The chronic effects of cannabis on memory in humans: a review. Curr Drug Abuse Rev 1: 81-98.

Takahashi RN, Singer G (1979). Self-administration of delta 9-tetrahydrocannabinol by rats. Pharmacol Biochem Behav 11: 737-740.

Torregrossa MM, Gordon J, Taylor JR (2013). Double dissociation between the anterior cingulate cortex and nucleus accumbens core in encoding the context versus the content of pavlovian cocaine cue extinction. J Neurosci 33: 8370-8377.

Torregrossa MM, Kalivas PW (2008). Neurotensin in the ventral pallidum increases extracellular gamma-aminobutyric acid and differentially affects cue- and cocaine-primed reinstatement. J Pharmacol Exp Ther 325: 556-566.

Torregrossa MM, Sanchez H, Taylor JR (2010). D-cycloserine reduces the context specificity of pavlovian extinction of cocaine cues through actions in the nucleus accumbens. J Neurosci 30: 10526-10533.

Verrico CD, Gu H, Peterson ML, Sampson AR, Lewis DA (2014). Repeated $\Delta 9$-tetrahydrocannabinol exposure in adolescent monkeys: persistent effects selective for spatial working memory. Am J Psychiatry 171: 416-425.

Verrico CD, Liu S, Bitler EJ, Gu H, Sampson AR, Bradberry CW et al (2012). Delay- and dose-dependent effects of $\Delta^{9}$-tetrahydrocannabinol administration on spatial and object working memory tasks in adolescent rhesus monkeys. Neuropsychopharmacology 37: 1357-1366.

Wiley JL, Burston JJ (2010). Chronic Delta9-tetrahydrocannabinol during adolescence increases sensitivity to subsequent cannabinoid effects in delayed nonmatch-to-position in rats. Pharmacol Biochem Behav 94: 516-523.

Zawilska JB, Wojcieszak J (2014). Spice/K2 drugs-more than innocent substitutes for marijuana. Int J Neuropsychopharmacol 17: $509-525$. 